\title{
A H9N2 Human Case and Surveillance of Avian Influenza Viruses in Live Poultry Markets - Huizhou City, Guangdong Province, China, 2021
}

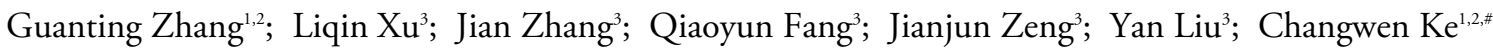

\section{Summary}

What is already known about this topic?

An increasing number of human infected avian influenza A (H9N2) cases have been reported. In 2021, 11 human infections with influenza $A$ virus subtype H9N2 (A/H9N2) have been reported in China.

What is added by this report?

A new case of H9N2 that occurred in April 2021 in Huizhou City, Guangdong Province, China, was reported in this study. Epidemiological and laboratory information of the case and routine influenza surveillance data of avian influenza A were presented in this report.

What are the implications for public health practice?

The emergence of a human infected with Avian Influenza Virus H9N2 demonstrates that there is an urgent need to strengthen the surveillance of influenzalike illness and live poultry market.

Since the first human infection of low pathogenicity avian influenza A (H9N2) virus was detected in Hong Kong, China in 1999 (1), this strain has been reported to intermittently infect humans. In April 2021, a case of H9N2 was reported in Huizhou City, Guangdong Province, China. The patient was sampled by throat swabs, which were sent to Huizhou CDC for quantitative reverse transcription-polymerase chain reaction (RT-PCR) testing. An investigation group carried out epidemiological and environmental investigations to find other avian influenza cases.

\section{INVESTIGATION AND RESULTS}

Emergency sampling of environment was conducted; both the viral RNA from the throat swab of the patient and environment were extracted using the QIAamp ${ }^{\circledR}$ Viral RNA Mini Kit (Qiagen, Germany) according to the manufacturer's instructions, and
RT-PCR assays with specific primers and probe sets were used for detecting the avian influenza A virus(H9N2) M gene. The virus gene segments were obtained through metagenomic sequencing. Genome sequences were aligned with the reference genomes downloaded from GenBank using the basic local alignment search tool (BLAST). The avian influenza virus activity in live poultry markets in Huizhou was analyzed using IBM SPSS Statistics for Windows (version 22.0. Armonk, NY: IBM Corp, USA).

The case occurred in a 30-year-old female living in Huicheng District of Huizhou City. On April 12, 2021, she experienced symptoms of sore throat but no fever. After medical treatment, her symptoms were alleviated. On April 20, 2021, she had symptoms of fever with an auxiliary temperature of $38.5^{\circ} \mathrm{C}$ and was admitted to the Huizhou Central People's Hospital for treatment. Her respiratory symptoms were paroxysmal cough, mainly dry cough, and wheezing. Other symptoms included chills, headache, and general malaise. The blood routine examination confirmed that the absolute value of neutrophils increased and that of lymphocytes decreased. The patient refused Xray check as she thought it was unnecessary due to her perceived mild symptoms. On April 21, the patient's throat swabs were collected and sent to the Huizhou CDC as a sample for routine influenza surveillance. On April 23, she was found to be positive for H9N2.

The patient had a history of travel to Dali Bai Minority Region, Yunnan Province from April 14 to 18 but denied having any direct contact history with live birds or other poultry. Photographs indicated that the patient did not wear a mask as required in a park. Before she went to Yunnan Province, she left her car in a surface parking lot, which was surrounded by many trees and birds. Her son was in a kindergarten near a live poultry market and was picked up by his grandmother. Their shoes may have been contaminated by sewage, poultry manure, and feathers when they passed through the live poultry market. 
Through RT-PCR assays, the cycle threshold value (Ct value) of the patient's throat swab was 32, which indicated the viral load of the sample was fairly low. As a result, the 8 sequences of $\mathrm{H} 9 \mathrm{~N} 2$ were unobtainable and only 9 gene reads were obtained (2 HA gene reads, 2 NA gene reads, 4 PB2 gene reads, $1 \mathrm{M}$ gene reads), with the length being $50 \mathrm{bp}$ for each read. The BLAST results showed that these gene reads had high homology with chicken H9N2 strains isolated from Guangdong Province, Zhejiang Province, and Shandong Province. The molecular characterization of the gene reads was analyzed, and the amino acid residues in proteins of these gene reads were the same or similar to reference strains $\mathrm{A} /$ chicken/China/ GD2021/2021(H9N2) and A/Zhongshan/201501/ 2015(H9N2), but different to reference strain A/chicken/Hong Kong/G9/1997(H9N2) as shown in the Table 1. A total of 40 environment samples of the live poultry market near the patient's son's kindergarten were collected. The nucleic acid positive rate of avian influenza A virus was detected, the $\mathrm{H}$ 9 subtype positive rate was 50\% (20/40), but H5 and H7 subtype were not detected.

A total of 386 environment samples were collected in poultry markets in Huizhou. The nucleic acid positivity rate of avian influenza A was detected in 176 of these samples from December 2020 to August 2021. The nucleic acid positive rate of subtype $\mathrm{H} 9$ fluctuated between $30.00 \%$ and $65.38 \%$ among different months, without significant difference $\left(\chi^{2}=56.00\right.$, $P>0.05$ ) (Figure 1). The highest positivity rate of avian influenza A virus was in August 2021, and the lowest was in April 2021.

\section{DISCUSSION}

So far in 2021, 11 human infections with A/H9N2 have been reported (2-3). Among them, Guangdong Province reported four cases, Yunfu City reported two cases, Meizhou City and Huizhou City each reported one case (4-5). According to the routine influenza surveillance of Huizhou City, avian influenza A virus

TABLE 1. The molecular characteristics of the H9N2 gene reads from Huizhou City.

\begin{tabular}{|c|c|c|c|c|c|c|c|c|c|}
\hline \multirow{2}{*}{ Virus } & \multicolumn{2}{|c|}{ HA } & \multicolumn{2}{|c|}{ NA } & \multicolumn{5}{|c|}{ PB2 } \\
\hline & $334^{*}$ & 501 & 432 & 434 & 299 & 327 & 334 & 336 & 381 \\
\hline A/chicken/Hong Kong/G9/1997(H9N2) & $A$ & $\mathrm{~K}$ & $\mathrm{~K}$ & $\mathrm{~T}$ & $\mathrm{~K}$ & G & $\mathrm{K}$ & $\mathrm{F}$ & M \\
\hline A/chicken/China/GD2021/2021(H9N2) & $S$ & Q & $\mathrm{H}$ & $\mathrm{P}$ & $\mathrm{R}$ & G & $S$ & $S$ & $\mathrm{~L}$ \\
\hline A/Zhongshan/201501/2015(H9N2) & $S$ & Q & Q & $\mathrm{P}$ & $\mathrm{R}$ & G & $S$ & $S$ & $\mathrm{~L}$ \\
\hline The H9N2 gene reads from Huizhou City & $\mathrm{F}$ & $Q$ & $\mathrm{H}$ & $P$ & $\mathrm{R}$ & $\mathrm{C}$ & $S$ & $S$ & $\mathrm{~L}$ \\
\hline
\end{tabular}

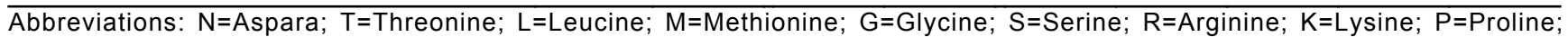
$\mathrm{F}=$ Phenylalanine.

*The mutation sites found in the detected 9 gene reads.

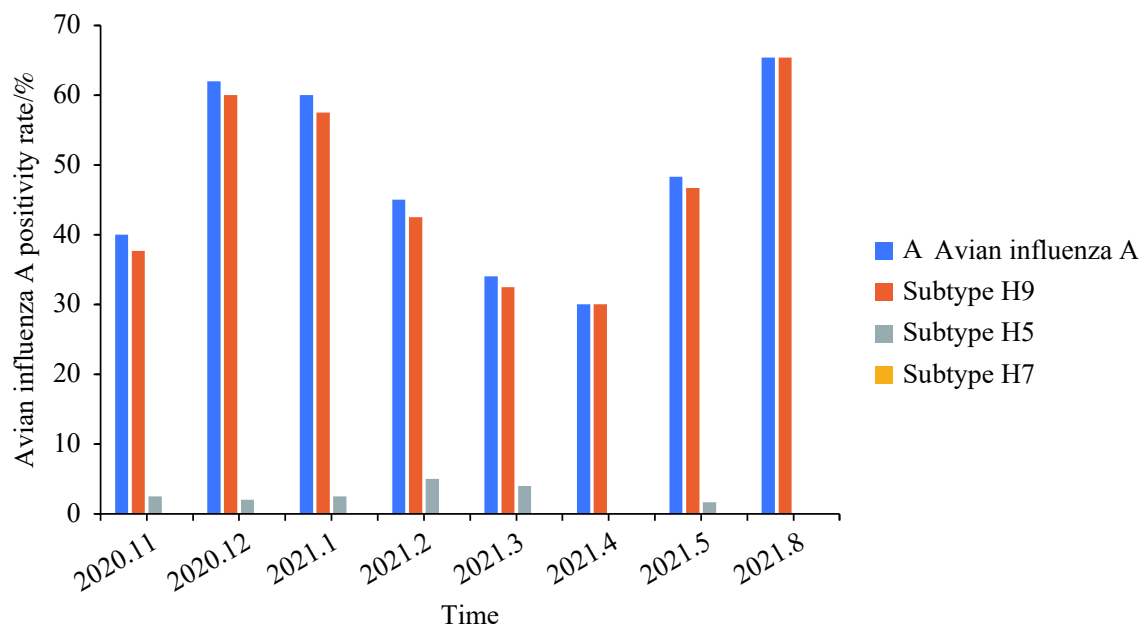

FIGURE 1. The monthly distribution of avian influenza A virus positive rate in Huizhou City.

Note: The subtype H7 AIV was undetected among the monitoring period; The subtype H5 AIV was undetected in April 2021 and August 2021. 
had a high positivity rate in poultry markets with subtype H9 being the most common subtype of avian influenza virus in poultry. H9 had a high positivity rate, while other subtypes were relatively rare.

Molecular characteristics analysis suggested that the risk of human infection was not higher than that of previous $\mathrm{H} 9 \mathrm{~N} 2$ avian influenza viruses. Combined with the epidemiological investigation, clinical symptoms of the patient, and the surveillance of avian influenza in the live poultry market, it is believed that the cause of the disease was mainly associated with exposure to the market that was contaminated by H9N2 avian influenza viruses. Therefore, she was likely infected by inhaling aerosols containing viruses (G).

New cases of human infection with avian influenza have been constantly occurring in China ( 7 ). There have also been several outbreaks of human infection with $\mathrm{H} 5 \mathrm{~N} 6$ and $\mathrm{H} 7 \mathrm{~N} 9$ avian influenza in Huizhou City (8). It is important to prevent avian influenza outbreaks by understanding the risks of avian influenza virus infection using detailed epidemiological investigation of patients and emergency environmental monitoring. Enhanced environmental monitoring of live poultry market is helpful to fully understand the contamination situation of avian influenza virus in the whole province. Therefore, closing the live poultry market and enhancing publicity and education in a timely manner are essential due to the high positivity rate of $\mathrm{H} 9 \mathrm{~N} 2$ in environmental samples. This case highlights a weakness of influenza surveillance in humans. Since the reported H9N2 case just had mild clinical symptoms and was found through influenzalike illness (ILI) surveillance, it is recommended that routine ILI surveillance be improved by increasing the sample number and frequency of sampling. It is also important to carry out surveillance in the population who work in the live poultry markets and poultry farms.

The findings in this report were subject to some limitations. First, the viral load of the sample was fairly low, so 8 gene sequences of H9N2 could not be obtained. Second, although emergency sampling of environment was conducted, bird populations in the live poultry market were not stable, so infected poultry may have been missed. Third, environment samples in Yunnan Province could not be obtained, so the relationship between travel history and $\mathrm{H} 9 \mathrm{~N} 2$ infection could not be excluded.

Conflicts of interest: No conflicts of interest declared.

doi: $10.46234 / \mathrm{ccdcw} 2021.273$

\# Corresponding author: Changwen Ke, kecw1965@aliyun.com.

\begin{abstract}
School of Public Health, Sun Yat-sen University, Guangzhou, Guangdong, China; ${ }^{2}$ Guangdong Provincial Center for Disease Control and Prevention, Guangzhou, Guangdong, China; ${ }^{3}$ Huizhou Municipal Center for Disease Control and Prevention, Huizhou, Guangdong, China.
\end{abstract}

Submitted: October 10, 2021; Accepted: November 30, 2021

\section{REFERENCES}

1. Peiris M, Yuen KY, Leung CW, Chan KH, Mmrcp PI, FRCPath RL, et al. Human infection with influenza H9N2. Lancet 1999;354(9182): 916 - 7. http://dx.doi.org/10.1016/S0140-6736(99)03311-5.

2. World Health Organization. Influenza at the human-animal interface summary and assessment, 12 February 2019. https://www.who.int/ publications $/ \mathrm{m} /$ item/influenza-at-the-human-animal-interface-summaryand-assessment-12-february-2019. [2021-8-12].

3. World Health Organization. Influenza at the human-animal interface summary and assessment, 13 December 2018. https://www.who.int/ publications $/ \mathrm{m} /$ item/influenza-at-the-human-animal-interface-summaryand-assessment-13-december-2018. [2021-8-12].

4. World Health Organization. Influenza at the human-animal interface summary and assessment, 22 June 2021. https://www.who.int/ publications $/ \mathrm{m} /$ item/influenza-at-the-human-animal-interface-summaryand-assessment-22-june-2021. [2021-8-12].

5. GISAID. EpiCoV ${ }^{\mathrm{rm}}$ Database. https://db.cngb.org/gisaid/. [2021-9-17]. (In Chinese).

6. Li S, Zhou YF, Gao SJ, Pang QH, Miao ZM. A small-scale study on airborne transmission of H9N2 avian influenza virus under field conditions. J Infect Dev Ctries 2018;11(12):962 - 6. http://dx.doi.org/ $10.3855 /$ jidc. 9013

7. Wang RP, Jiang YG, Zhao GM, Guo XQ, Michael E. Outbreak gold standard' selection to provide optimized threshold for infectious diseases early-alert based on China infectious disease automated-alert and response system. Curr Med Sci 2017;37(6):833 - 41. http://dx.doi.org/ 10.1007/s11596-017-1814-9.

8. Bao LL, Bi YH, Wong G, Qi WB, Li FD, Lv Q, et al. Diverse biological characteristics and varied virulence of $\mathrm{H} 7 \mathrm{~N} 9$ from Wave 5. Emerg Microbes Infect 2019;8(1):94 - 102. http://dx.doi.org/10.1080/ 22221751.2018 .1560234 